\title{
COMPARATIVE STUDY OF EFFECT OF SWERTIA CHIRATA LEAF EXTRACT ON INDINAVIR TREATED RATS
}

\author{
RAJESH CS ${ }^{1 *}$, RAJENDRA HOLLA ${ }^{2}$, VENKATESH PATIL ${ }^{1}$, ANAND AS ${ }^{3}$ \\ ${ }^{1}$ Department of Pharmacology, Navodaya Medical College Raichur, Karnataka, India. ${ }^{2}$ Department of Pharmacology, KSHEMA, Mangalore, \\ Karnataka, India. ${ }^{3}$ Department of Pathology, Navodaya Medical College Raichur, Karnataka, India. Email: rajeshsharma.c.s@gmail.com
}

Received: 17 October 2016, Revised and Accepted: 01 November 2016

\section{ABSTRACT}

Background: Indinavir is widely used for the treatment of human immunodeficiency virus (HIV) infection. It is known to cause hyperglycemia or insulin resistance and hyperlipidemia.

Aim and Objectives: To study the effect of Swertia chirata leaf extract with metformin and pioglitazone on indinavir treated rats.

Methods: Swiss albino rats were divided into five Groups of six animals each. All the groups (except control) were treated with indinavir $216 \mathrm{mg} / \mathrm{kg}$ (oral) for 15 days. Group I (control) received normal saline (oral) from day 1 to day 15 , Group II received indinavir $216 \mathrm{mg} / \mathrm{kg}$ (oral), Group III received S. chirata plant extract $500 \mathrm{mg} / \mathrm{kg}$ (oral) from day 8 to day 15, Group IV received pioglitazone $4 \mathrm{mg} / \mathrm{kg}$ (oral) from day 8 to day 15 , and Group V received metformin $180 \mathrm{mg} / \mathrm{kg}$ (oral) from day 8 to day 15. The biochemical parameters such as serum glucose, insulin, and lipid levels were measured on day 15. Results were analyzed using one-way analysis of variance followed by Bonferroni's multiple comparison test.

Results: Indinavir $(216 \mathrm{mg} / \mathrm{kg})$ treated rats showed a significant $(\mathrm{p}<0.05)$ increase in glucose and insulin levels and also altered lipid levels. This indicates indinavir produces diabetic-like state in rats. S. chirata extract (500 mg/kg) decreases glucose and insulin levels and also improves lipid levels the effect is almost similar to metformin and pioglitazone.

Conclusion: Indinavir causes elevated glucose, insulin and lipid levels, so care must be taken while prescribing indinavir for HIV patients. Treatment with S. chirata extract improved the altered glucose, insulin, and lipid profile in indinavir treated rats.

Key words: Indinavir, Insulin resistance, Diabetes dyslipidemia, Glucose intolerance.

(c) 2017 The Authors. Published by Innovare Academic Sciences Pvt Ltd. This is an open access article under the CC BY license (http://creativecommons. org/licenses/by/4. 0/) DOI: http://dx.doi.org/10.22159/ajpcr.2017.v10i2.15723

\section{INTRODUCTION}

Antiretroviral agents prevent the progression of disease which is caused by human immunodeficiency virus (HIV). Therapy includes a combination of minimum three drugs and available regimens forms more than 1100 combinations. The unique property of these drugs is need for lifelong administration to control virus replication and to prevent the development of drug resistance. The contribution of PI's is considered to a great extent in the prevention of HIV-associated morbidity and mortality over many years and remain a cornerstone of HAART. Long-term therapy with PI's is found to cause insulin resistance (IR) and hyperglycemia, epidemic Type-2 diabetes mellitus and other complications such as hyperglycemia, hypercholesterolemia or lipodystrophy. PI's found to produce long-term suppression of viremia, increase in CD4 lymphocyte counts, reduced disease progression, and decrease in mortality, the benefits and toxicity of this drug needs to be balanced [1-4].

This class of drug found to be very effective but they also found to be associated with metabolic changes that greatly increase lifetime risk $[5,6]$. Among these, one of the important metabolic changes is alteration of normal glucose-insulin homeostasis which remains particularly prevalent and alarming clinical change in affected patients. IR, impaired glucose tolerance, and Type-II diabetes mellitus are the most common in patients receiving HAART, especially with PI's [7-9].

The occurrence of DM is four-fold higher in HIV-infected patients receiving HAART compared to HIV-seronegative men [10]. Lifespan of HIV infected patients is increased due to availability advanced antiretroviral drugs. HIV-infected patients can lead a normal life but there is an increased chance of risk from noninfectious caused such as cardiovascular disease and diabetes [11-13].

Pioglitazone act by increasing insulin sensitivity in peripheral tissue and it increases glucose transport into muscle and adipose tissue. It also activates the genes that regulate fatty acid metabolism in peripheral tissue [14]. Metformin is known as insulin sensitizer; that is, it increases glucose uptake and utilization by target tissues, thereby decreasing IR. It requires insulin for its action but does not promote insulin secretion $[15,16]$.

Swertia chirata belongs to the family of Gentianaceae. It is also known as Chirata, Chirayata and this is also mentioned in Charaka Samhitha a classical Indian medical text. Based on research, scientists confirmed this plant as a potential antidiabetic agent. Recent decades researchers also revealed its antimalarial, anti-inflammatory, antioxidative, and anticarcinogenic properties of this plant. Hence, we have selected it for this study, the effect of $S$. chirata leaf extract with metformin and pioglitazone on indinavir-treated rats.

\section{METHODS}

\section{Raring of animals}

For this study male albino Wister rats, weighing between 230 and 300 g were used. Rats were procured from the central animal house of institution. The experiment protocol used for this study was approved by the institutional animal ethical committee [Ref.No: KSHEMA/ 
IAEC/03/2013]. They were maintained under standard laboratory condition at temperature $23 \pm 2{ }^{\circ} \mathrm{C}$, humidity $50 \pm 10 \%$ with $12 \mathrm{hrs}$ light/12 hrs dark cycles. Animals were maintained at polypropylene cages, rat pellets and water were given ad libitum.

\section{Drugs and chemicals}

Indinavir was obtained from yarrow chem products, Mumbai. Metformin and Pioglitazone were obtained from Mahalakshmi chemicals, Hyderabad. Ketamine injection was obtained from Neon Laboratories Limited, Mumbai.

\section{Reagents and kits}

The biochemical parameters such as glucose and lipid profile were measured using commercially available kits (Agappe Diagnostics Ltd, Kochi). To measure insulin levels the Ultra sensitive Rat insulin enzymelinked immunosorbent assay (ELISA) kit were obtained from Genxbio Health Sciences Private Limited, New Delhi.

\section{Preparation of $S$. chirata leaf extract}

Leaves of $S$. chirata were used for this study; leaves were dried at room temperature. Dried leaves are taken into a Soxhlet apparatus; ethanol is used for the process of extraction.

\section{Phytochemical analysis}

S. chirata ethanolic extract was subjected to preliminary phytochemical analysis for detection of major chemical constituents such as alkaloids, phenols, resins, tannins, terpenoid, and saponins.

\section{Experimental design}

The animals were divided into five Groups $(n=6)$. The study was conducted for 15 days. A pilot study was conducted initially to determine dose of indinavir which causes hyperglycemia or IR and hyperlipidemia. At the same time, dose of antidiabetic drugs such as metformin and pioglitazone was determined.

Group I (control) received normal saline (oral) from day 1 to day 15 , Group II received indinavir $216 \mathrm{mg} / \mathrm{kg}$ (oral), Group III received S. chirata plant extract $500 \mathrm{mg} / \mathrm{kg}$ (oral) from day 8 to day 15 , Group IV received pioglitazone $4 \mathrm{mg} / \mathrm{kg}$ (oral) from day 8 to day 15 , and Group V received metformin $180 \mathrm{mg} / \mathrm{kg}$ (oral) from day 8 to day 15. All the groups (Except control) were treated with indinavir $216 \mathrm{mg} / \mathrm{kg}$ (oral) for 15 days. The $2 \%$ Gum acacia were used as a vehicle.

At the end of study period, i.e., on $15^{\text {th }}$ day, rats were anesthetized and fasting blood glucose was collected by retro-orbital sinus puncture for estimation of biochemical parameters.

\section{Biochemical estimation}

Blood samples were centrifuged at 2000 RPM and serum was separated. The serum was used for the biochemical investigations. Serum glucose levels were measured by glucose oxidase and peroxidase (GOD-POD) method, triglycerides measured by glycerol phosphate oxidase-POD, total cholesterol (CH), high-density lipoproteins (HDL), and lowdensity lipoproteins (LDL)-CH by oxidase POD (CHOD-PAP) methods. Very LDL (VLDL) CH was estimated using the formulas, VLDL=TG/5. The serum insulin levels were estimated by ELISA method using ELISA reader.

\section{Oral glucose tolerance test (OGTT)}

At the end of experimental period, OGTT was performed in all groups of rats. The $12 \mathrm{hr}$ fasted rats were treated with glucose solution at a dose of $2 \mathrm{~g} / \mathrm{kg}$ body weight by oral route, followed by collection of blood sample for analysis.

\section{Statistical analysis}

The data were presented in mean \pm standard error of the mean. Results were analyzed using one-way analysis of variance followed by Bonferroni's multiple comparison test. $\mathrm{p}<0.05$ was considered to be significant.

\section{RESULT}

Phytochemical analysis

The preliminary phytochemical analysis of $S$. chirata leaf extract showed the presence of Carbohydrate, Coumarins and Tannin.

\section{Biochemical parameters}

In Group II rats treated with indinavir $216 \mathrm{mg} / \mathrm{kg}$ (oral) significantly increased Insulin, total CH, TG's, LDL, and VLDL levels without causing much change in HDL levels in comparison to control group. It indicates that indinavir produces diabetic-like state in rats. Treatment of rats in Group III with ethanolic extract of $S$. chirata leaf extract significantly reduced the elevated insulin, total $\mathrm{CH}$, TG's, LDL, and VLDL levels in comparison Group II. There was no significant difference on insulin and lipid profile between the standard drugs Metformin, pioglitazone and with the treatment of $S$. chirata leaf extract on rats, as shown in Fig. 1 and Table 1

\section{OGTT}

It was observed that after a 2 hrs glucose load Group II shows significant high serum glucose levels compared to normal. In Groups III and V, the glucose levels were significantly less compared to Group II (Fig. 2).

Random serum glucose concentration was measured which shows a significant difference compared to control and treatment groups (Fig. 3).

\section{DISCUSSION}

Protease inhibitors are known to induce a syndrome of IR along with that it may also cause hyperglycemia, hyperlipidemia, and fat

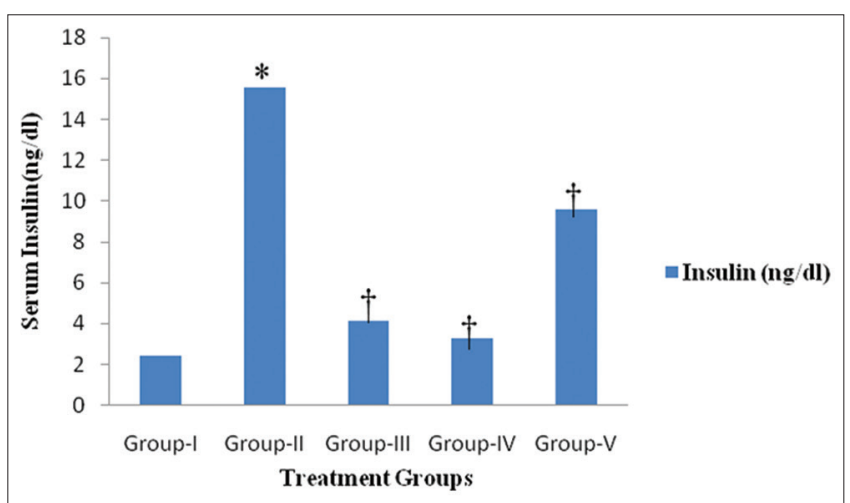

Fig. 1: 1 Effect of indinavir, Swertia chirata extract and standard drugs on rat serum insulin levels. Values are expressed in mean \pm standard error of the mean, ${ }^{*} \mathbf{p}<0.05$ vs. Normal control, tp $<0.05$ vs. diabetic control

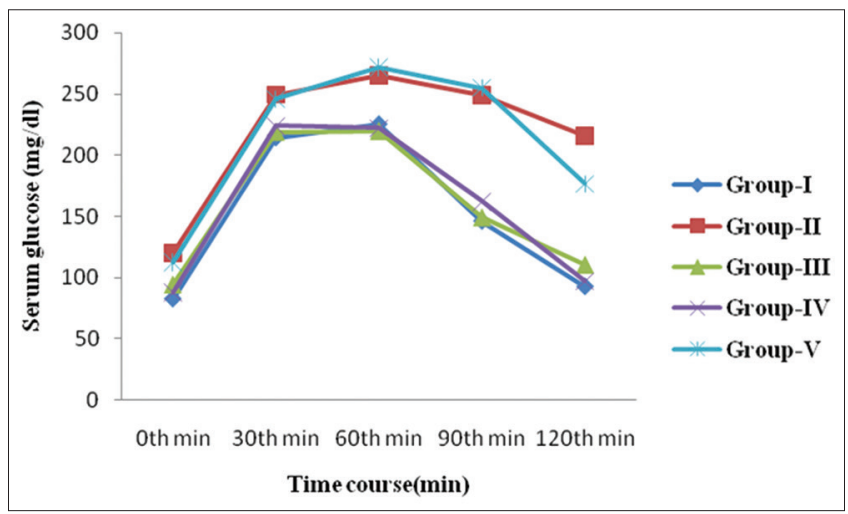

Fig. 2: Effect of indinavir, Swertia chirata extract and standard drugs on rat serum glucose levels 
Table 1: Effect of indinavir, Swertia chirata extract, and standard drugs on rat lipid profile

\begin{tabular}{|c|c|c|c|c|c|}
\hline Groups & TG (mg/dl) & TC (mg/dl) & HDL (mg/dl) & VLDL (mg/dl) & LDL (mg/dl) \\
\hline Group-I & $72.5 \pm 2.74$ & $96.6 \pm 2.1$ & $40.0 \pm 2.3$ & $14.5 \pm 0.5$ & $42.1 \pm 3.8$ \\
\hline Group-II & $219.3 \pm 13.4^{*}$ & $144.6 \pm 7.1^{*}$ & $30.8 \pm 2.2$ & $43.8 \pm 2.6^{*}$ & $69.9 \pm 8.2^{*}$ \\
\hline Group-III & $79.6 \pm 2.6 \dagger$ & $98.5 \pm 2.1 \dagger$ & $35.3 \pm 2.4$ & $15.9 \pm 0.5 \dagger$ & $47.2 \pm 3.9$ \\
\hline Group-IV & $86.1 \pm 3.9 \dagger$ & $98.1 \pm 4.6 \dagger$ & $37.0 \pm 1.5$ & $17.2 \pm 0.7 \dagger$ & $43.9 \pm 5.0$ \\
\hline Group-V & $145.8 \pm 22.1 \dagger$ & $109.02 \pm 6.1 \dagger$ & $31.6 \pm 1.7$ & $29.1 \pm 4.4 \dagger$ & $48.2 \pm 7.9$ \\
\hline
\end{tabular}

Values are expressed in mean \pm SEM, ${ }^{*} p<0.05$ vs. Normal control, $\uparrow \mathrm{p}<0.05$ vs. diabetic control, HDL: High density lipoproteins, VLDL: Very low density lipoproteins,

LDL: Low density lipoproteins, S. chirata: Swertia chirata, SEM: Standard error of the mean

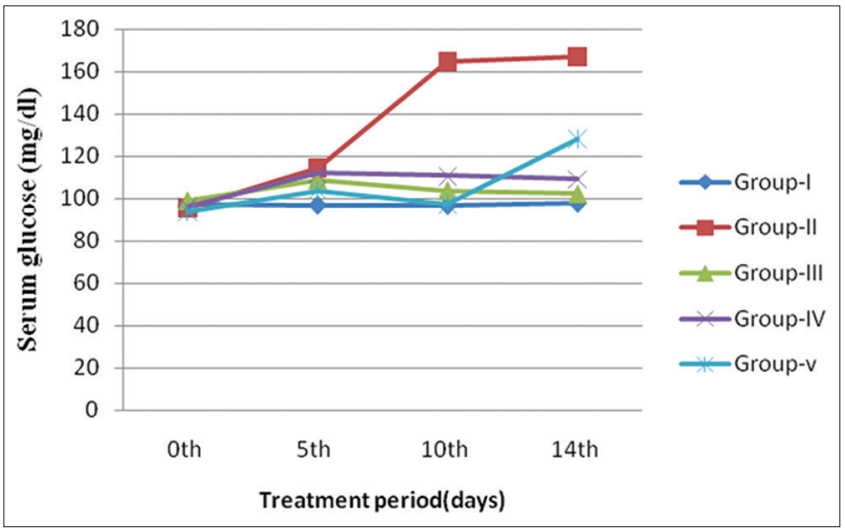

Fig. 3: Effect of indinavir, Swertia chirata extract and standard drugs on rat serum glucose levels

redistribution. It resembles the metabolic syndrome with high risk of cardiovascular diseases. Many research studies were done to assess IR using a homeostasis model assessment of IR (HOMA-IR). The calculated score higher than four using HOMA-IR indicates IR. Early studies of insulin profile in the patients infected with HIV infection were no IR or hyperglycemia was observed, but clamp studies indicated the increased IR. Protease inhibitors can cause decrease in insulin-stimulated glucose transport in fat cells [16]. Patients with HIV infection must be screened at the beginning of HAART therapy, and later during the treatment. Certain professionals suggest for fasting blood glucose as a screening tool; others recommend OGTT which should be performed as a part of screening procedures [17]

The actual mechanism of IR is not well understood. IR occurs when normal insulin levels are no sufficient to stimulate glucose uptake in the insulin signaling pathway in insulin-sensitive tissues such as muscle, liver, and subcutaneous adipose tissue. Following manifestations such as hyperinsulinemia, hyperglycemia, and dislipidemia (hypertriglyceridemia) are seen in patients with protease inhibitors $[18,19]$.

The treatment with PIs caused partial lipodystrophies in some reports and also found to cause DM or insulin resistance along with higher triglyceride and $\mathrm{CH}$ levels. There is also peripheral fat wasting, accumulation of intra-abdominal fat has been observed in some patients. Inhibition of hormone sensitive lipase and insulin induced stimulation of lipoprotein lipase would result in net increase of fat. Inhibition of lipogenesis and stimulation of lipolysis results in inappropriate increase in free fatty acids. Increased availability of free fatty acids would further increase hepatic triglyceride synthesis and increases hepatic glucose output, which causes compensatory increase in insulin secretion to maintain glucose uptake homeostasis. There are many theories suggests that PIs therapy induces DM, beside that there is increasing evidence that there is an increase in insulin levels suggesting emergence of IR [20,21].

It has been observed that PIs selectively inhibit GLUT4 activity in vitro, which provides an important mechanism for the development of IR observed in the patients with HIV infection. Whole body glucose disposal takes place by glucose transport, GLUT4 is expressed in tissue responsible for glucose disposal (skeletal/cardiac muscle and fat), and it is believed to be principal transporter for mediating insulin stimulated glucose uptake at these sites. PIs causes inhibition of GLUT4, and the inhibition is reversible when the treatment is stopped. PIs inhibitors induced IR also associated with lipodystrophy syndrome caused by GLUT4 inhibition. Chronic treatment of indinavir causes elevated free fatty acids secondarily contribute to IR, prolonged treatment may cause redistribution of fat and hypertriglyceridemia may also cause IR. Hyperinsulinism from GLUT4 inhibition may contribute to fat distribution $[22,23]$

S. chirata was known to possess antidiabetic activity since a long time. To study the hypoglycemic and antidiabetic role of $S$. chirata 95\% ethanolic extract and other four fractions were used, and the significant effect was produced by lowering blood sugar level [24]. The Swertia extract significantly decreases the $\mathrm{CH}$ and lipid levels. Phytochemical analysis was performed and the extract was known to contain carbohydrate, coumarins, and Tannin. The S. chirata is also known to contain three main phytochemicals amarogentin, mangiferin, and Swertia merin. Mangiferin is known to have blood glucose lowering property and also shows suppressive effect on lipid profile $[25,26]$.

\section{CONCLUSION}

This study reveals that $S$. chiratais is effective against indinavir-induced hyperglycemia or IR and hyperlipidemia. $S$. chirata with its multiple beneficial effects would seem to be useful as an adjuvant with indinavir (protease inhibitor) on AIDS patients.

\section{REFERENCES}

1. Brunton LL, Lazo JS, Parker KL. Antiretroviral agents and treatment of HIV infection. Goodman and Gilman's The Pharmacological Basis of Therapeutics. 11 $1^{\text {th }}$ ed. New York: McGraw-Hill Companies; 2006. p. $1613-45$.

2. Sharma HL, Sharma KK. HIV and antiretroviral drugs. Principles of Pharmacology. $1^{\text {st }}$ ed. New Delhi: Paras Medical Publisher; 2007. p. $809-20$.

3. Dubé MP, Johnson DL, Currier JS, Leedom JM. Protease inhibitorassociated hyperglycaemia. Lancet 1997;350(9079):713-4.

4. Noor MA, Lo JC, Mulligan K, Schwarz JM, Halvorsen RA, Schambelan M, et al. Metabolic effects of indinavir in healthy HIVseronegative men. AIDS 2001;15(7):F11-8.

5. Schwarz JM, Lee GA, Park S, Noor MA, Lee J, Wen M, et al. Indinavir increases glucose production in healthy HIV-negative men. AIDS 2004;18(13):1852-4.

6. Nolan D. Metabolic complications associated with HIV protease inhibitor therapy. Drugs 2003;63(23):2555-74.

7. Dubé MP. Disorders of glucose metabolism in patients infected with human immunodeficiency virus. Clin Infect Dis 2000;31(6):1467-75.

8. Tsiodras S, Mantzoros C, Hammer S, Samore M. Effects of protease inhibitors on hyperglycemia, hyperlipidemia, and lipodystrophy: A 5-year cohort study. Arch Intern Med 2000;160(13):2050-6.

9. Mastan SK, Kumar EK. Relative potency of protease inhibitors on glucose-insulin homeostasis, hemoglobin and glycosylated hemoglobin in normal rats. Der Pharm Lett 2009;1(1):108-16.

10. Brown TT, Cole SR, Li X, Kingsley LA, Palella FJ, Riddler SA, et al. Antiretroviral therapy and the prevalence and incidence of diabetes mellitus in the multicenter AIDS cohort study. Arch Intern Med 2005; 165(10):1179-84 
11. Palella FJ Jr, Delaney KM, Moorman AC, Loveless MO, Fuhrer J, Satten GA, et al. Declining morbidity and mortality among patients with advanced human immunodeficiency virus infection. HIV Outpatient Study Investigators. N Engl J Med 1998;338(13):853-60.

12. Green ML. Evaluation and management of dyslipidemia in patients with HIV infection. J Gen Intern Med 2002;17(10):797-810.

13. Justman JE, Benning L, DanoffA, Minkoff H, Levine A, Greenblatt RM, et al. Protease inhibitor use and the incidence of diabetes mellitus in a large cohort of HIV-infected women. J Acquir Immune Defic Syndr 2003;32(3):298-302.

14. El Gawly HW, Tawfik MK, Rashwan ME, Baruzaig AS. The effect of pioglitazone on the liver of streptozotocin-induced diabetic albino Wistar rats. Eur Rev Med Pharmacol Sci 2009;13(6):443-51.

15. Cheng JT, Huang CC, Liu IM, Tzeng TF, Chang CJ. Novel mechanism for plasma glucose-lowering action of metformin in streptozotocininduced diabetic rats. Diabetes 2006;55(3):819-25.

16. Grunfeld C. Insulin resistance in HIV infection: Drugs, host responses or restoration to health. Int AIDS Soc USA 2008;16(2):89-94.

17. Kalra S, Kalra B, Agrawal N, Unnikrishnan A. Understanding diabetes in patients with HIV/AIDS. Diabetol Metab Syndr 2011;3(1):2.

18. Ismail WI, Pillay TS. Insulin resistance induced by antiretroviral drugs: Current understanding of molecular mechanisms. JEMDSA 2009;14(3):129-32.

19. Viganò A, Brambilla P, Pattarino G, Stucchi S, Fasan S, Raimondi C, et al. Long-term evaluation of glucose homeostasis in a cohort of
HAART-treated HIV-infected children: A longitudinal, observational cohort study. Clin Drug Investig 2009;29(2):101-9.

20. Gómez-Vera J, de Alarcón A, Jiménez-Mejías ME, Acosta D, Prados D, Viciana P. Hyperglycemia associated with protease inhibitors in HIV-1infected patients. Clin Microbiol Infect Dis 2000;6(7):389-93.

21. Maurice HB, Kiula GI, Kitundu MN. Diabetes And HIV/AIDS: Molecular interaction between insulin receptor and protease inhibitors that leads to diabetes in people taking ARVS. TAJONAS 2010;1(1):51-6.

22. Hruz PW, Murata H, Qiu H, Mueckler M. Indinavir induces acute and reversible peripheral insulin resistance in rats. Diabetes 2002;51(4):937-42.

23. Choi YH, Lee MG, Lee I. Effects of diabetes mellitus induced by alloxan on the pharmacokinetics of metformin in rats: Restoration of pharmacokinetic parameters to the control state by insulin treatment. J Pharm Pharm Sci 2008;11(1):88-103.

24. Sekar BC, Mukherjee B, Chakravarti RB, Mukherjee SK. Effect of different fractions of Swertia chirayita on the blood sugar level of albino rats. J Ethnopharmacol 1987;21(2):175-81.

25. Kavitha KN, Dattari AN. Experimental evaluation of antidiabetic activity of Swertia chirata aquous extract. J Public Health Med Res 2013;1(2):71-5.

26. Rafatullah S, Tariq M, Mossa JS, al-Yahya MA, al-Said MS, Ageel AM. Protective effect of Swertia chirata against indomethacin and other ulcerogenic agent-induced gastric ulcers. Drugs Exp Clin Res $1993 ; 19(2): 69-73$ 